\title{
NÚCLEOS EXÓTICOS E SÍNTESE DOS ELEMENTOS QUÍMICOS
}

\author{
Nilton Teruya* \\ Departamento de Física, Universidade Federal da Paraíba, Campus I, CP 5008, 58051-970 João Pessoa - PB, Brasil \\ Sérgio Barbosa Duarte \\ Centro Brasileiro de Pesquisas Físicas, Rua Dr. Xavier Sigaud, 150, 22290-180 Rio de Janeiro - RJ, Brasil
}

Recebido em 2/2/11; aceito em 3/8/11; publicado na web em 26/9/11

\begin{abstract}
EXOTIC NUCLEI AND SYNTHESIS OF CHEMICAL ELEMENTS. We have around ninety chemical elements available in nature, which were produced mainly by nuclear reactions inside stars. The fusion reactions are the main synthesis process which generates the light and intermediate masses elements. The synthesis begins with the hydrogen burning reaching the region of iron mass nuclei. Heavier elements are synthesized by neutron capture processes, forming exotic nuclei with large neutron excess. These systems present characteristics very different from nuclei inside of stable atoms; they only occur in particular astrophysical environments or are produced artificially in special laboratory conditions. This work discusses some properties of the exotic nuclei and how they participate in the synthesis of elements.
\end{abstract}

Keywords: chemical element; exotic nuclei; nucleosynthesis.

\section{INTRODUÇÃO}

A descoberta da radioatividade e a identificação da carga elementar do elétron, no final do século XIX, deram início a uma nova fase nas investigações da estrutura mais fundamental da matéria, mostrando indícios de que o átomo não é uma partícula indivísivel. O modelo atômico hoje vigente, com elétrons envolvendo um pequeno núcleo pesado e positivamente carregado, foi evidenciado experimentalmente por E. Rutherford em 1911, no célebre trabalho onde discutiu os dados experimentais de espalhamentos de partículas alfa obtidos por G. Geiger e E. Marsden. ${ }^{1,2}$ Este modelo do átomo nuclear está completando 100 anos, colocado como uma ideia inovadora, necessitou de novas teorias para fundamentá-lo, estabelecendo desafios que conduziram à formulação da mecânica quântica, que ainda dava os primeiros passos. Apesar de demonstrada a existência do núcleo atômico, a sua composição só ficou determinada em 1932, com a descoberta do nêutron por J. Chadwick. Hoje sabemos que este núcleo atômico é composto por prótons e nêutrons, partículas que interagem atrativamente pela intensa força nuclear e são olhados como diferentes estados de uma mesma partícula, o "núcleon". A carga elétrica positiva do núcleo é dada pelos prótons e a quantidade destas partículas define o número atômico. Átomos de mesmo número atômico, mas que diferem na quantidade de nêutrons, são chamados isótopos e são diferentes formas com se apresenta um dado elemento químico. ${ }^{3} \mathrm{O}$ termo nuclídeo é usado para designar determinado isótopo de um elemento químico, cujo núcleo tem " $\mathrm{Z}$ " prótons e " $\mathrm{N}$ " nêutrons, num total de "A" núcleons (com $\mathrm{A}=\mathrm{Z}+\mathrm{N}$ ).

A teoria acerca da estrutura atômica da matéria ficou bem estabelecida e hoje é bastante difundida e a ideia do átomo com elétrons envolvendo um núcleo já é introduzida no ensino de Química e Física a nível da educação fundamental.

A proposta do presente artigo é discutir aspectos gerais do núcleo atômico, apresentando algumas noções sobre a estrutura e estabilidade nuclear, e discutindo as participações decisivas das espécies nucleares exóticas na síntese e na variedade dos elementos químicos existentes. Estas informações podem contribuir para levar um pouco desses conhecimentos para as pessoas interessadas no assunto e

\footnotetext{
*e-mail: nteruya@ fisica.ufpb.br
}

buscam complementar os conteúdos de Química e Física ensinados ao nível da graduação.

\section{VARIEDADE E ABUNDÂNCIA DOS ELEMENTOS}

A variedade de substâncias que compõem o mundo que nos cerca se origina da combinação dos elementos químicos, os quais comparecem na natureza em diferentes abundâncias. Esta grandeza é uma medida da quantidade relativa dos elementos, sendo obtida pela observação, no sistema solar, dos meteoritos e da análise espectroscópica das estrelas. Notamos que os elementos químicos que usualmente conhecemos se apresentam com isótopos estáveis e outros radioativos; os estáveis são mais abundantes porque não decaem, enquanto que os radiativos vão se desintegrando e se transmutando para outros mais estáveis. Por exemplo, o hidrogênio possui dois isótopos estáveis: o prótio (com apenas um próton no núcleo) e o deutério (com um próton e um nêutron no núcleo), sendo que o prótio é o mais abundante (abundância de 99,985\%).

A abundância dos elementos químicos na natureza está relacionada com a estabilidade e a massa dos nuclídeos que, por seu turno, dependem diretamente da proporção entre os números de prótons e nêutrons que os compõem. Dentre os elementos conhecidos, o hidrogênio $(Z=1)$ é o mais simples e abundante, respondendo por aproximadamente $75 \%$ da massa total do universo massivo conhecido. A partir dele é que os outros são sintetizados. O segundo elemento é o hélio $(Z=2)$ que ao lado do hidrogênio somam juntos quase $99 \%$ de toda massa dos elementos do universo, ficando a mínima fatia de pouco mais de $1 \%$ para ser compartilhada entre cerca de uma centena de outros elementos. Encontramos 90 elementos químicos naturais, desde o hidrogênio até o urânio $(Z=92)$, outros elementos são muito instáveis ou pesados demais, não existem ou se encontram em pouca quantidade na natureza, como o tecnécio $(Z=43)$, o promécio $(Z=61)$ e os elementos de número atômico além do urânio, como o plutônio $(Z=94)$. Recentemente, os dois elementos de maiores números atômicos encontrados artificialmente, $Z=117$ e 118, tiveram suas detecções consolidadas em experimentos. ${ }^{4}$ Entretanto, já foram observados em torno de 3200 nuclídeos com meias-vidas grandes o suficinte para que algumas de suas propriedades sejam medidas. Meiavida é uma das grandezas físicas diretamente associadas à estabilidade 
nuclear e fornece uma medida do tempo necessário para que metade da quantidade inicial de núcleos radioativos sofra decaimentos. Os elementos químicos estáveis estão limitados ao número atômico $\mathrm{Z}=$ 83 (bismuto), dos quais 81 têm pelo menos um isótopo estável. Estes elementos contribuem com aproximadamente 262 nuclídeos estáveis (considerando-se como estáveis aqueles de meia-vida maior do que $1 \times 10^{17}$ anos), distribuídos da seguinte forma: ${ }^{5} 155$ têm número de prótons e nêutrons par ( $\mathrm{Z}$ e N pares), 102 com um deles par ( $\mathrm{Z}$ ou N par), apenas 5 com os dois ímpar ( $\mathrm{Z}$ e $\mathrm{N}$ ímpares).

Núcleos com grande diferença entre os números de prótons e nêutrons são bastante instáveis e apresentam características muito diferentes dos estáveis, daí serem classificados como núcleos exóticos. ${ }^{6}$ Estes tipos nucleares não são encontrados na natureza, para estudá-los, precisam ser produzidos artificialmente em laboratório. As pesquisas sobre estes núcleos vêm mostrando efeitos novos da estrutura nuclear, servindo para colocar à prova e reformular muito dos conceitos já estabelecidos.

Os elementos muito leves como hidrogênio, hélio e lítio têm suas formações em boa parte justificada por uma síntese primordial na história do universo. Em grande parte, estes elementos foram sintetizados nos primeiros momentos da evolução do universo numa nucleossíntese cosmológica. Os de massas intermediárias são produzidos em estrelas massivas, que conseguem às custas de contrações gravitacionais aquecer o seu interior o suficiente para desencadear processos de fusão de novos elementos a partir dos mais leves por elas produzidos. As etapas da produção dos elementos são acompanhadas por alterações na estrutura da estrela e nas condições de seu interior (temperatura e pressão) que, dependendo da massa da estrela, podem ou não permitir o prosseguimento da cadeia de produção dos elementos de massas intermediárias $(\mathrm{A}<56) .{ }^{7}$ Resta-nos saber sobre a síntese dos elementos pesados, aqueles além do ferro e, mesmo, os transurânicos.

As abordagens correntes discutidas nos livros didáticos para a síntese dos elementos enfocam as reações de fusão nuclear que ocorrem nas estrelas. Porém, estes processos se limitam à produção dos elementos até a região de massa do ferro; para além deste, outros mecanismos respondem pela síntese dos elementos mais pesados. As pesquisas nestes temas são atuais e passam pela discussão de sistemas nucleares exóticos que só recentemente puderam ser produzidos e estudados mais detalhadamente nos laboratórios. A participação dos núcleos exóticos na síntese dos elementos pesados pode surgir principalmente no final da vida ativa de estrelas muito massivas, assim como em nucleossíntese explosiva na fase de supernova destas estrelas. Nestes eventos são lançados ao espaço grande parte dos elementos pesados que foram sintetizados. Este material recicla aquele já presente no espaço e é usado para fazer novos objetos, como estrelas, planetas, asteroides, ou qualquer outro objeto astrofísico material.

\section{ESTABILIDADE NUCLEAR E A ENERGIA DE LIGAÇÃO DOS NÚCLEOS}

A estabilidade nuclear relaciona-se com a capacidade do núcleo manter a sua estrutura natural por longo tempo, ou resistindo a algum estímulo externo que induza sua transmutação. Ao contrário, núcleos radioativos são instáveis, decaem espontaneamente emitindo algum tipo de radiação que os transformam em outros núcleos mais estáveis. Núcleos estáveis têm meias-vidas muito longas (várias dezenas de bilhões de anos), já os radioativos podem ter meias-vidas de frações de segundos, embora alguns deles decaiam lentamente com meiasvidas de até milhões ou bilhões de anos (a Tabela 1 apresenta alguns valores de meias-vidas). Na Tabela 2 estão mostrados alguns tipos de radiações emitidas por núcleos instáveis, com os respectivos núcleos resultantes do decaimento.
Tabela 1. Alguns exemplos de núcleos estáveis e instáveis com suas meiasvidas. Os tipos de radiação são explicados na Tabela 2

\begin{tabular}{lll}
\hline Nuclídeo & Tipo de instabilidade & Meia-vida \\
\hline${ }_{1}^{1} \mathrm{H}_{0}$ & Estável & \\
${ }_{2}^{3} \mathrm{H}_{1}$ & Beta menos & 12,3 anos \\
${ }_{2}^{4} \mathrm{He}_{2}$ & Estável & \\
${ }_{3}^{5} \mathrm{Li}_{2}$ & 1 próton & $\sim 10^{-22} \mathrm{~s}$ \\
${ }_{3}^{10} \mathrm{Li}_{7}$ & 1 nêutron & $\sim 10^{-22} \mathrm{~s}$ \\
${ }_{3}^{11} \mathrm{Li}_{8}$ & Beta menos & $\sim 8,5 \mathrm{~ms}$ \\
${ }_{4}^{8} \mathrm{Be}_{4}$ & Desintegra-se em 2 alfas & $\sim 10^{-16} \mathrm{~s}$ \\
${ }_{6}^{14} \mathrm{C}_{8}$ & Beta menos & $\sim 5730$ anos \\
${ }_{25}^{45} \mathrm{Fe}_{19}$ & 2 prótons & $\sim 3 \mathrm{~ms}$ \\
${ }^{130}{ }_{48} \mathrm{Cd}_{82}$ & Beta menos & $\sim 0,2 \mathrm{~s}$ \\
${ }_{61}^{149}{ }_{69} \mathrm{Tm}_{78}$ & 1 próton & $\sim 0,6 \mathrm{~s}$ \\
${ }_{82}^{208} \mathrm{~Pb}_{126}$ & Estável & \\
${ }_{238}^{238} \mathrm{U}_{146}$ & Alfa & $\sim 4,5$ bilhões de anos \\
\hline
\end{tabular}

Tabela 2. O núcleo pai ${ }_{Z}^{A} X_{N}$ decai com a emissão de um dos tipos de radiação. O número total de núcleons e a carga elétrica total devem ser iguais antes e depois do decaimento. O núcleo residual $\mathrm{Y}$, ou núcleo filho, é determinado de acordo com a radiação, se houver mudanças na carga elétrica, há transmutação do elemento

\begin{tabular}{|c|c|c|}
\hline Símbolo & Tipo da radiação emitida & Núcleo filho \\
\hline$\alpha$ & $\begin{array}{l}X \rightarrow Y+\alpha \\
\text { A partícula alfa é um núcleo do átomo de hélio, } \\
\text { contém } 2 \text { prótons e } 2 \text { nêutrons. }\end{array}$ & ${ }_{Z-2}^{A-4} Y_{N-2}$ \\
\hline$\beta$ & $\begin{array}{l}X \rightarrow Y+e^{-}+\bar{v}_{e}: \text { decaimento beta menos, um } \\
\text { nêutron do núcleo decai num próton e há } \\
\text { emissão de um elétron e um antineutrino. }\end{array}$ & ${ }_{Z+1}^{A} Y_{N-1}$ \\
\hline$\beta^{+}$ & $\begin{array}{l}X \rightarrow Y+e^{+}+v_{e} \text { : decaimento beta mais, um } \\
\text { próton do núcleo decai num nêutron, há emissão } \\
\text { de um pósitron e um neutrino. }\end{array}$ & ${ }_{Z-1}^{A} Y_{N+1}$ \\
\hline$\gamma$ & $\begin{array}{l}X^{*} \rightarrow X+\gamma \\
\text { Radiação gama, emissão de fótons, partícula } \\
\text { sem massa ou carga elétrica. }\end{array}$ & ${ }_{Z}^{A} X_{N}$ \\
\hline $\mathrm{n}$ & $\begin{array}{l}X^{*} \rightarrow X+n \\
\text { Emissão de um nêutron. }\end{array}$ & ${ }_{Z}^{A-1} X_{N-1}$ \\
\hline $\mathrm{p}$ & $\begin{array}{l}X^{*} \rightarrow Y+p \\
\text { Emissão de um próton. }\end{array}$ & ${ }_{Z-1}^{A-1} Y_{N}$ \\
\hline
\end{tabular}

A radiação pode envolver emissão de partículas ou ondas eletromagnéticas, saindo diretamente de um estado nuclear instável para outro mais estável, ou sequencialmente, formando diferentes sistemas intermediários até chegar à configuração estável final.

Um exemplo de emissão sequencial é a radioatividade alfa (Tabela 2) dos isótopos na região do urânio $(Z=92)$, que decai passando de um elemento a outro, até alcançar a região de massa do chumbo ( $\mathrm{Z}$ = 82) ou do bismuto $(Z=83)$, os elementos estáveis mais pesados encontrados na natureza. Há previsões teóricas sobre a existência de uma ilha de estabilidade na região de massa dos elementos superpesados em torno do número atômico $\mathrm{Z}=114,{ }^{8}$ mas que ainda não foi completamente estabelecida. No outro extremo, novos sistemas exóticos, como o isótopo ${ }^{12} \mathrm{O}$ do oxigênio, têm deficiência em nêutrons e apresentam emissão espontânea de dois prótons, ${ }^{9}$ no $\mathrm{O}^{12}$ pode haver decaimento sequencial através do nitrogênio-11, ou diretamente para o carbono- 10.

Evidências experimentais mostram que quando prótons ou 
nêutrons, ou ambos, são em número par, eles ficam mais fortemente ligados do que quando há um núcleon desemparelhado. Há uma forma de energia de emparelhamento que contribui para aumentar a energia de ligação do núcleo. Outras evidências indicam que, semelhante aos átomos dos gases nobres, existem números mágicos nucleares: $2,8,20,28,50$ e 82; alcançando o 126 no caso de nêutrons. Dentro do núcleo os prótons e os nêutrons se distribuem numa estrutura de camadas de energia, os núcleos com números de prótons ou nêutrons mágicos têm suas estruturas de camadas completas e são mais difíceis de serem excitados, sendo particularmente mais ligados e mais estáveis do que os seus vizinhos de número de massa.

A energia de ligação nuclear é uma quantidade de energia que foi dispendida no processo de formação e estruturação do núcleo. De fato, o que acontece é que a massa do núcleo é menor do que a soma das massas dos seus núcleons individuais, a diferença é convertida na energia de ligação. A transformação de massa em energia é explicada pela famosa relação de Einstein: $E=\Delta m c^{2}$, onde $\Delta m$ é a diferença de massa (a quantidade que diminui na construção do núcleo) e $c$ a velocidade da luz ( $c=300 \mathrm{mil} \mathrm{km} / \mathrm{s}$ no vácuo).

A energia de ligação representada pelo símbolo $B(Z, A)$ é uma medida da energia requerida para quebrar o núcleo em seus constituintes nucleônicos. De outro modo, para arrancar uma partícula do núcleo precisa-se fornecer uma quantidade mínima de energia para vencer a sua ligação com o núcleo, definida como energia de separação. Para os núcleos naturalmente radioativos, as partículas da radiação não ficam ligadas e escapam espontaneamente do núcleo. Porém, deve-se observar que a estabilidade nuclear e a energia de ligação dos núcleons dependem também de fatores relacionados com a estrutura do núcleo.

O comportamento médio da energia de ligação por núcleon, representado com o símbolo $B(Z, A) / A$, em função do número de massa “ $A$ ” está apresentado na Figura 1.

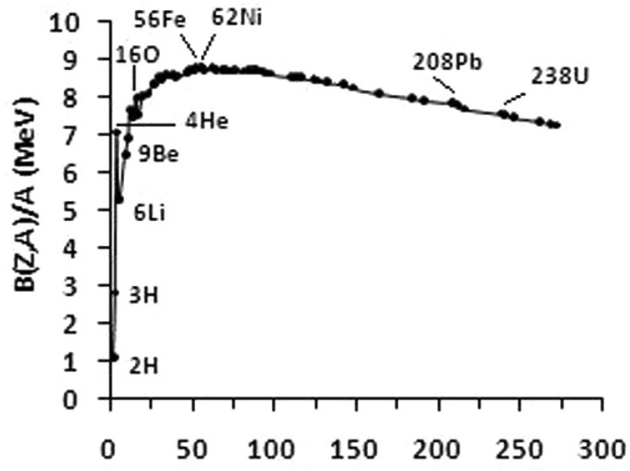

A

Figura 1. Energia média de ligação por núcleon " $B(Z, A) / A$ ” de alguns nuclídeos. A linha contínua é para mostrar um comportamento médio. As energias foram calculadas a partir dos dados da ref. 5

Nesta figura se observa que o aumento de "A" leva a um rápido crescimento da energia de ligação média por núcleon. Isto ocorre porque o caráter atrativo da força nuclear ainda não alcançou uma saturação. Após este rápido crescimento inicial, a curva " $B(Z, A) / A$ " alcança um valor máximo para núcleos na região de massa do ferro e do níquel, a seguir, a linha decresce lentamente, mesmo para valores crescentes de "A". Este comportamento se deve à distribuição de matéria no núcleo e ao curto alcance da força nuclear que leva a uma saturação (interação apenas com a vizinhança próxima). Isto também se deve ao fato de que a força elétrica de repulsão entre os prótons contribui significativamente para esta diminuição lenta de
" $B(Z, A) / A$ " na região dos núcleos pesados, $\mathrm{A}>56$. Na radioatividade natural os núcleos emitem radiação espontaneamente, mas núcleos estáveis também podem se tornar instáveis quando são induzidos artificialmente por reações nucleares.

\section{Núcleos exóticos e a assimetria entre números de nêutrons e prótons}

Esta categoria de núcleos apresenta grande assimetria entre os números de prótons e nêutrons, as suas características fogem às regras gerais que se aplicam aos núcleos estáveis, daí porque são classificados como núcleos exóticos.

Para entender as características gerais dos núcleos exóticos, vamos retomar a discussão sobre a energia de ligação.

Outro efeito importante na ligação nuclear é o da assimetria entre os números de prótons e nêutrons. Conforme os elementos ficam mais pesados, aumenta o número de prótons e, consequentemente, a repulsão coulombiana entre eles também aumenta. Para contrabalançar este efeito e estabilizar o núcleo, o número de nêutrons cresce mais rápido do que o de prótons, já que os nêutrons não têm carga elétrica e a força nuclear é atrativa. Os nuclídeos estáveis até aproximadamente a região do cálcio $(Z=20)$ têm números de prótons e nêutrons aproximadamente iguais, as variações em torno de $\mathrm{Z}=\mathrm{N}$ são pequenas, mas à medida que os elementos ficam mais pesados, há grande desvio em favor do excesso de nêutrons (Figura 2).

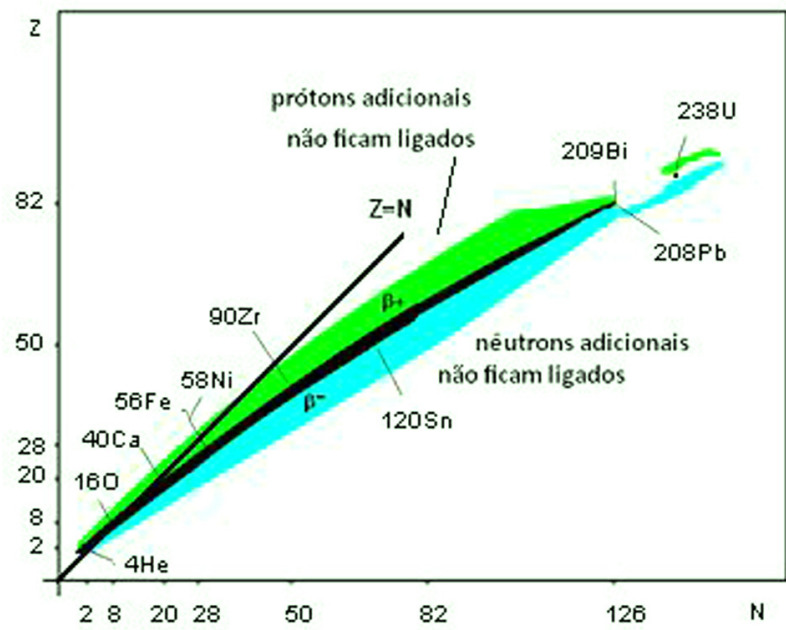

Figura 2. Esquema aproximado da distribuição dos núcleos em relação às suas estabilidades (com base nos dados da ref. 5). Os números marcados nos eixos são mágicos. Na região central escura estão os núcleos estáveis ou com meia-vida acima de dezenas de milhões de anos; a cor verde representa a região da instabilidade beta mais; em azul a instabilidade beta menos. Para adiante destas regiões, há emissão de núcleons (ou emissores alfa), os excessos de nêutrons ou de prótons são tão grandes que os nêutrons ou prótons adicionais não ficam ligados. Alguns nuclídeos mais conhecidos, e com números mágicos de prótons elou nêutrons (exceto $o^{238} U$ ), estão em destaque, assinalando a região de maior estabilidade nuclear. A linha $Z=N$ serve para referência dos nuclídeos com números iguais de prótons e nêutrons

Apesar da necessidade do excesso de nêutrons para estabilizar núcleos de elementos pesados, os isótopos de qualquer elemento tendem a se desestabilizar quando o excesso de nêutrons se torna excessivo. Há um balanço mais apropriado entre os números de prótons e nêutrons para cada elemento, sendo este um dos fatores que determina o isótopo mais estável. Desta forma, o crescente enriquecimento de nêutrons, numa cadeia isotópica, começa a produzir isótopos com instabilidade beta menos. $\mathrm{O}$ motivo disso é que 
a radiação beta menos faz o núcleo se transmutar num isótopo de outro elemento químico, com uma unidade a mais no número atômico (Tabela 2). Esta instabilidade acontece no sentido de diminuir a diferença nêutron-próton em busca de maior estabilidade. A partir daí, a continuidade do enriquecimento pode alcançar um limite onde os nêutrons adicionais não ficam mais ligados e escapam, atingindo o limiar da separação de nêutrons.

Para comparação do tamanho do excesso de nêutrons nos núcleos exóticos, um dos núcleos mais pesado e estável encontrado na natureza é o chumbo-208 $\left({ }_{82}^{208} \mathrm{~Pb}_{126}\right)$, que apresenta vários estados excitados ligados e uma razão entre os números de nêutrons e prótons $N / Z=1,5$, enquanto que um dos núcleos exóticos mais estudados, ${ }^{10} \mathrm{O}$ lítio-11 $\left({ }_{3}^{11} \mathrm{Li}_{8}\right)$, tem $N / Z=2,7$ e nenhum estado excitado ligado. Um estado excitado é alcançado quando o núcleo absorve energia e sai do seu estado normal, e é dito ligado quando os seus núcleons ainda permanecem presos no núcleo. Outro dado comparativo bastante peculiar é que o tamanho do ${ }_{3}^{11} \mathrm{Li}_{8}$ (com 11 núcleons) é bem próximo ao do ${ }_{82}^{208} \mathrm{~Pb}_{126}$ (com 208 núcleons), sendo esta uma característica tão marcante que classificou este tipo de núcleo como exótico.

Os núcleos exóticos são altamente instáveis e têm um tempo de vida curto. A meia-vida do ${ }_{3}^{11} \mathrm{Li}_{8}$ é aproximadamente $8 \mathrm{~ms}$, transmutase no berílio-11 $\left({ }_{4}^{11} \mathrm{Be}_{7}\right)$ via decaimento $\beta^{-}$. Outro efeito decorrente do aumento do excesso de nêutrons é que há uma tendência destes núcleos em apresentar alterações na sequência dos números mágicos. ${ }^{11}$ Um exemplo disso é o oxigênio-24 $\left({ }_{8}^{24} \mathrm{O}_{16}\right)$, cuja estrutura sugere uma alteração do número mágico 20 para 16.

Em primeira aproximação os núcleos ricos em nêutrons podem ser descritos por uma configuração onde um caroço, correspondendo a um isótopo mais estável do elemento, está envolto pelos nêutrons do excesso. A pequena energia de ligação dos nêutrons de valência possibilita que eles fiquem distribuídos numa extensão espacial muito além daquela ocupada pelo caroço, contribuindo para a composição de uma nuvem bastante difusa que contém o excesso de nêutrons, formando um "halo" de nêutrons. Como exemplo, no caso do ${ }_{3}^{11} \mathrm{Li}_{8}$, o núcleo caroço é o ${ }_{3}^{9} \mathrm{Li}_{6}$, e o halo é formado pelos dois nêutrons de valência. Na Figura 3 são apresentadas as energias de separação de 1 e 2 nêutrons dos isótopos do lítio. Enquanto a energia de ligação de 1 nêutron sofre as flutuações discutidas anteriormente devido aos efeitos da energia de emparelhamento, o comportamento da energia de dois nêutrons é apenas decrescente, o isótopo ${ }^{11} \mathrm{Li}$ é um limite para esta ligação. Energia de separação negativa significa que

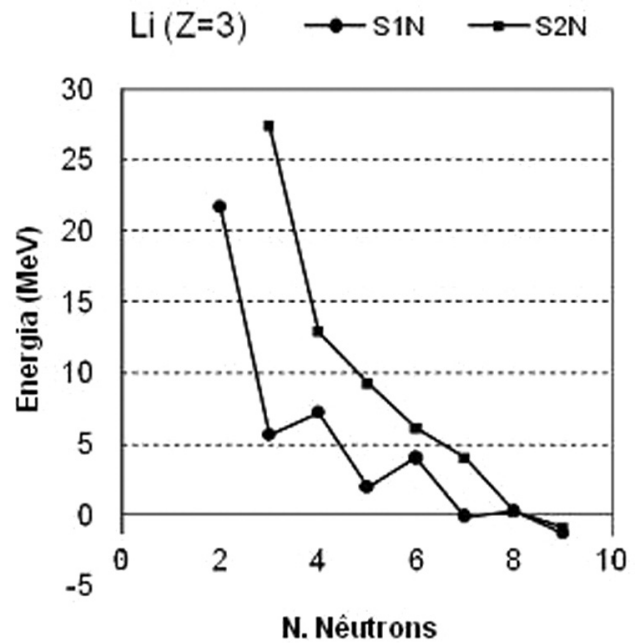

Figura 3. Energias de separação $S_{1 N} e S_{2 N}$, de 1 e 2 nêutrons, respectivamente, para os isótopos do lítio. Valores negativos indicam que os nêutrons não estão ligados no núcleo o nêutron não fica ligado e escapa do núcleo. Estas características mostram outro aspecto interessante deste núcleo, enquanto no ${ }^{10} \mathrm{Li}$ o nêutron extra ao núcleo caroço não fica ligado e escapa, os dois nêutrons de valência ficam presos no ${ }^{11} \mathrm{Li}$, um efeito devido à energia de emparelhamento. Assim, se for retirado qualquer nêutron do halo no ${ }^{11} \mathrm{Li}$, o sistema se divide em três partes, caroço mais dois nêutrons. Núcleos deste tipo, cujo caroço não prende um núcleon extra, mas prende dois, são chamados borromeanos, ${ }^{12}$ uma referência à união representada pelos três anéis entrelaçados no símbolo heráldico da família italiana renascentista Borromeo: se qualquer dos anéis for retirado, os outros se desatam.

Outra consequência importante da fraca ligação dos nêutrons do halo é que estes núcleos podem ser facilmente excitados, num modo de vibração onde os nêutrons do excesso oscilam contra o caroço mais coeso. ${ }^{13}$ Este modo de vibração, chamado ressonância pigmeia, está localizado em baixa energia de excitação, mas acima do limiar de emissão de nêutrons, o que faz dele um importante mecanismo de excitação e emissão de nêutrons.

Uma questão curiosa é a de como são obtidas as informações que levam a formular as teorias do átomo e do núcleo. Mas, o que chega a ser até mesmo intrigante é como obter estas informações de objetos que vivem tão pouco tempo. A discussão deste assunto é ampla, mas aqui vamos fazê-la de forma sucinta. Uma maneira de sondar estes sistemas minúsculos é fazendo um projétil apropriado incidir sobre um alvo feito do material cuja estrutura dos núcleos se quer conhecer, detectores apropriados são posicionados para registrar os eventos que ocorrem quando o projétil e o alvo interagem. Os registros constituem os dados da experiência, que passam por análises e dão subsídios para formulação de teorias sobre a estrutura do núcleo em observação, uma técnica bastante refinada de exploração do objeto desconhecido. No caso dos núcleos exóticos isso é um tanto mais delicado, devido às suas pequenas meias-vidas. Neste caso, os experimentos são realizados com estes núcleos no papel de projétil, fazendo-os incidir em alvos de materiais estáveis de estrutura conhecida. Em uma das técnicas, o feixe exótico é produzido como resultado de uma reação primária, onde determinados nuclídeos e alvos são previamente escolhidos para fornecer o feixe de núcleos exóticos que se quer estudar. Vários produtos de reação saem deste primeiro estágio. Um espectrômetro de massa, sistema composto pela aplicação de campos elétricos e magnéticos, seleciona o feixe exótico. A seguir, este feixe é direcionado para impactar o segundo alvo, de onde novos fragmentos de reação são produzidos e fornecem os dados para serem analisados. A Figura 4 apresenta um esquema geral de um método utilizado nestes estudos. A produção e análise de experimentos com feixes de núcleos exóticos hoje é desenvolvida em pelo menos uma dúzia de laboratórios espalhados pela América do Norte, Europa e Japão. Em nosso país temos o RIBRAS (Radioactive Ions Beams in Brazil), um sistema de produção de feixes radioativos que está em operação desde Fevereiro de 2004 no Instituto de Física da Universidade de São Paulo.

\section{O PAPEL DOS NÚCLEOS EXÓTICOS NA SÍNTESE DE ELEMENTOS PESADOS}

Um requisito para a estrela produzir elementos e continuar em sua jornada evolutiva é que as reações nucleares que proporcionam sua evolução devem liberar energia. Para que as reações nucleares aconteçam, as condições físicas nas estrelas, como temperatura, densidade dos núcleos reagentes e pressão, devem alcançar valores característicos que propiciem as ocorrências dessas reações. A pressão e densidade governam a probabilidade de os núcleos colidirem, quanto maior a pressão, mais aumentam as chances de colisões. A energia cinética dos núcleos aumenta para temperaturas mais elevadas, o que 


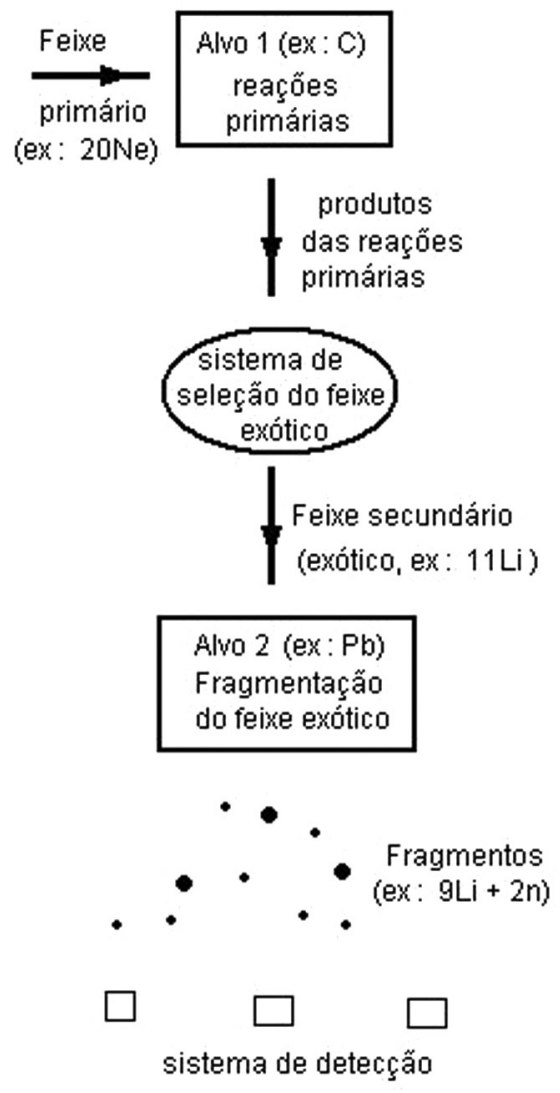

Figura 4. Esquema simplificado de um sistema de produção de feixes radiativos exótico em montagens experimentais. Cada um destes blocos ou seções inclui equipamentos variados, contendo complexos sistemas eletrônicos para acompanhamento da qualidade do feixe em diferentes setores. Os tipos de núcleos e fragmentos nos exemplos são alguns dos citados na literatura

permite que colisões entre núcleos vençam a barreira coulombiana e estes entrem em fusão efetivamente.

Na primeira fase da nucleossíntese nas estrelas, acontece a queima de hidrogênio com a consequente produção de hélio e energia, como ocorre no Sol. As estrelas passam a maior parte do tempo nesta fase de produção, enquanto estão na sequência principal do estágio da sua evolução. A produção de núcleos leves por captura de prótons e partículas alfa esbarra no problema da falta de elementos estáveis com massas $\mathrm{A}=5$ e $\mathrm{A}=8$. Com isso, a passagem do hélio para o carbono é bastante importante, pois implica na passagem por um gargalo, onde o processo de produção é bastante delicado. O nuclídeo berílio-8, que viria da fusão de duas alfas, é altamente instável (Tabela 1), mas se uma terceira alfa chegar antes de o berílio- 8 decair, há chances da produção de carbono-12. Caso estes eventos ocorram, a probabilidade de se formar o carbono-12 é aumentada graças a um estado excitado deste carbono numa energia próxima às dos sistemas berílio- 8 mais alfa ou de três alfas. Há também possibilidades de rotas alternativas que passam pela produção de núcleos exóticos para chegar ao carbono. A partir da produção do carbono, outras capturas alfas levam à produção de oxigênio e demais elementos.

Um processo de grande importância para a evolução da estrela e a produção dos elementos é o ciclo CNO (carbono - nitrogênio - oxigênio), proposto por $\mathrm{H}$. Bethe e, independentemente, por C. F. Weiszacker, na década de $1930 .^{7}$ Nesta cadeia, há consumo de quatro prótons com a produção de um núcleo hélio-4, o ponto chave do ciclo é que há liberação de energia preservando a quantidade de carbono-12 usada no ciclo.

Depois de ultrapassados os gargalos da falta de estabilidade em
$\mathrm{A}=5$ e $\mathrm{A}=8$, a síntese dos elementos por reações de fusão pode continuar até a região de massa do ferro e do níquel, em torno de A $=56$, conforme a massa da estrela. A partir daí surge outra barreira por causa do alto consumo de energia requerido nas reações de fusão. Isto ocorre devido à intensa repulsão coulombiana entre núcleos com grande quantidade de prótons, a energia necessária para a fusão é grande e inviabiliza a síntese de elementos pesados por estes processos. Este comportamento pode ser observado na Figura 1. A curva $B(Z, A) / A$ mostra que a fusão nuclear com liberação de energia ocorre para núcleos situados abaixo da região de massa do ferro, acima dele, são as reações de fissão que liberam energia. ${ }^{7,14}$

Os elementos com massa além do ferro e do níquel são produzidos por outros processos, principalmente pela captura de nêutrons. A Figura 5 mostra um esquema geral da sequência de reações envolvidas no processo. As primeiras ideias sobre a síntese dos elementos em ambientes ricos em nêutrons foram apresentadas na década de 1940, principalmente nos trabalhos de G. Gamow, R. Alpher e R. Herman, em modelos de nucleossíntese primordial. ${ }^{15}$ Porém, em 1957 o trabalho de Burbidge-Burbidge-Fowler-Hoyle $\left(\mathrm{B}^{2} \mathrm{FH}\right)$ introduziu alguns processos para a síntese dos elementos pesados nas estrelas; ${ }^{: 6}$ duas destas propostas apontavam para a formação de núcleos ricos em nêutrons por meio de capturas rápidas ou lentas de nêutrons. Em nosso país, os primeiros trabalhos desenvolvidos sobre este tema surgiram no final da década de 1970, no Centro Brasileiro de Pesquisas Físicas. ${ }^{17}$ Estes modos de produção dos elementos pesados estão bem estabelecidos atualmente, embora ainda persistam questões em aberto sobre as condições físicas, dos sítios astrofísicos onde ocorrem e sobre as propriedades dos núcleos ricos em nêutrons formados, os núcleos exóticos envolvidos. A estrutura dos núcleos é de fundamental importância nestes processos, uma vez que devem ser consideradas as taxas de captura de nêutrons, os modos de excitação nuclear e as formas de decaimento.

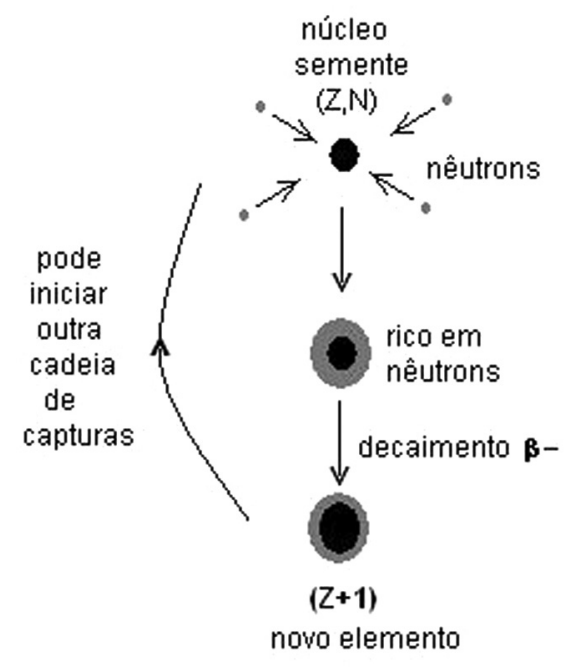

Figura 5. Esquema geral de uma cadeia de enriquecimento de nêutrons. A partir de um núcleo semente, a sequência de absorção de nêutrons leva à formação de isótopos cada vez mais ricos em nêutrons e, independente do processo, chega-se num ponto onde ocorre o decaimento beta menos, um novo elemento é produzido e uma nova cadeia pode ter sequência

Além das condições de temperatura, pressão e densidade de nêutrons na estrela, a evolução de uma cadeia de núcleos enriquecidos por nêutrons é definida pela competição entre as meias-vidas dos isótopos e o tempo que levam para capturar um nêutron do ambiente da estrela.

Os processos rápido e lento de captura de nêutrons ocorrem em escalas de tempo bem diferentes:18 o lento é o processo-s ("s" de slow), contrapondo-se ao rápido que é o processo-r ("r" de rapid). A 
lentidão ou a rapidez da captura é comparada com o tempo envolvido no decaimento beta menos dos núcleos enriquecidos. Cada processo necessita de determinadas condições ambientais compatíveis com a sobrevivência das espécies nucleares presentes durante o processo. $\mathrm{O}$ estudo destes processos requer o conhecimento das propriedades dos núcleos ricos em nêutrons, discutidas na seção anterior.

Apesar das suas características particulares, numa descrição geral destes processos, temos um núcleo semente bombardeado por nêutrons do ambiente da estrela e iniciando uma sequência de capturas destas partículas. O excesso crescente de nêutrons leva os isótopos a ficarem cada vez mais instáveis, mas, enquanto há enriquecimento, a captura de nêutrons é mais rápida do que o tempo característico do decaimento beta menos. A situação de instabilidade vai se acentuando com o aumento do número de nêutrons, até alcançar determinado isótopo onde o decaimento beta menos ocorre mais rápido e interrompe a sequência de capturas. Um novo elemento é produzido e o número atômico aumenta de uma unidade. Outra cadeia de enriquecimento pode ser iniciada e sintetizar um novo elemento.

Avalia-se que no processo-s o ambiente da estrela seja de baixa densidade de nêutrons, estimada em torno de $10^{8}$ nêutrons $/ \mathrm{cm}^{3}$. Como os nêutrons estão esparsos no meio, a taxa de captura de nêutron pelos núcleos presentes é menor ou, no máximo, comparável à taxa de decaimento beta dos núcleos formados. Assim, os núcleos instáveis que se formam têm tempo de decair e o percurso do processo ascende em número atômico próximo ao vale de estabilidade, com pequenos deslocamentos para o lado dos núcleos ricos em nêutron. Esta síntese alcança núcleos pesados até a região de massa do bismuto e do chumbo, os últimos e mais pesados elementos estáveis. Para além destes, os elementos são fortemente instáveis pela emissão de partículas alfa e a cadeia de captura de nêutrons próxima à linha de estabilidade beta é interrompida.

No processo-r o fluxo de nêutrons deve ser tão intenso que se formam núcleos muito ricos em nêutrons em muito pouco tempo. Estas são situações típicas de eventos explosivos de supernova, as condições físicas extremas de densidade e temperatura, sendo responsáveis pela síntese da maioria dos elementos químicos acima da massa do ferro e de quase a totalidade dos elementos acima do bismuto.

A captura de nêutrons é rápida, formando-se isótopos muito ricos em nêutrons, chegando mesmo a alcançar a região onde os nêutrons não se ligam mais aos núcleos. Para um grande e rápido enriquecimento de nêutrons é necessário que no ambiente da estrela haja um fluxo de nêutrons térmicos suficiente, as condições internas da estrela são estimadas em densidade de nêutrons acima de $10^{20}$ nêutrons $/ \mathrm{cm}^{3}$ e temperatura maior do que $10^{9}{ }^{\circ} \mathrm{C} . .^{18}$

Como mencionado anteriormente, em determinada cadeia de isótopos ricos em nêutrons o aumento crescente do número de nêutrons diminui a energia de ligação dos nêutrons de valência; além disso, para número ímpar de nêutrons a energia de ligação do nêutron desemparelhado é menor, enquanto que a energia de separação de dois nêutrons é sempre decrescente (Figura 3).

Como os núcleos estão expostos à radiação gama do ambiente altamente aquecido, eles podem absorvê-la, caso a energia térmica seja suficiente para arrancar nêutrons, eles são desligados, deixando o núcleo residual novamente exposto às outras capturas de nêutrons. Disso resulta um ciclo de capturas e desligamentos de nêutrons:

$$
{ }_{\mathrm{Z}}^{\mathrm{A}} \mathrm{X}_{\mathrm{N}}+\mathrm{n} \rightarrow{ }^{\mathrm{A}+1} \mathrm{Z}_{\mathrm{N}+1} ;{ }^{\mathrm{A}+1}{ }_{\mathrm{Z}} \mathrm{X}_{\mathrm{N}+1}+\gamma \rightarrow{ }_{\mathrm{Z}}^{\mathrm{A}} \mathrm{X}_{\mathrm{N}}+\mathrm{n}
$$

Quando este ponto é atingido, há um equilíbrio entre absorção e emissão de nêutron para determinado isótopo de massa "A". Neste equilíbrio, o processo de captura atinge um "ponto de espera", onde a captura e a emissão do nêutron estão em franca competição. Quando esta condição é atingida, o núcleo demora um tempo neste estágio até que ocorra o decaimento $\beta^{-}$e um novo elemento seja sintetizado. A seguir, pode ter início nova sequência de capturas de nêutrons que, posteriormente, produzirá outro elemento.

\section{CONSIDERAÇÕES FINAIS}

Os processos de síntese dos elementos pesados via formação de núcleos ricos em nêutrons estão bem estabelecidos, muito embora ainda nos falte o conhecimento com maior precisão de algumas propriedades dos núcleos exóticos que podem ser formados e sobre as condições físicas dos sítios onde a síntese ocorre. A formação e abundância dos elementos químicos transurânicos presentes na natureza são diretamente relacionadas com as propriedades nucleares e a estabilidade dos núcleos exóticos envolvidos no processo de síntese destes elementos. Os núcleos exóticos com maiores meias-vidas, apesar de muito pequenas, funcionam como ponto de acumulação ao longo da cadeia isotópica e originam os picos de abundância relativa dos elementos formados. Um ponto interessante nesta análise é que enquanto a estabilidade nuclear determina a formação, abundância e variedade dos elementos químicos leves e de massas intermediárias na natureza, são os sistemas nucleares exóticos, altamente instáveis, que tornam possível a formação dos elementos pesados.

\section{REFERÊNCIAS}

1. Rutherford, E.; Philosophical Magazine 1911, 21, 669

2. Geiger, H.; Marsden, E.; Proceedings of the Royal Society 1909, 82, 495.

3. Tolentino, M.; Rocha-Filho, R. C.; Chagas, A. P.; Quim. Nova 1997, 20, 103; Oki, M. C. M.; Química Nova na Escola 2002, nº 26, 21.

4. Cartlidge, E.; "New element 117 discovered", disponível em http:// physicsworld.com/cws/article/news/42272, acessada em Janeiro 2011; Chalmers, M.; "Researchers discover element 118", disponível em http://physicsworld.com/cws/article/news/26190, acessada em Janeiro 2011.

5. Estes números podem mudar se forem encontrados outros nuclídeos estáveis novos e, também, podem diferir de outros resultados por causa da escolha da meia-vida limite (consideramos maior do que $1 \times 10^{17}$ anos). As informações foram obtidas a partir dos bancos de dados: The Lund/LBNL Nuclear Data Search, Version 2.0, February 1999 (Chu, S. Y. F.; Ekström, L. P.; Firestone, R. B.); Audi, G.; Bersillon, O; Blachot, J.; Wapstra, A. H.; Nuclear Physics A 2003, 729, 3.

6. Poskanzer, A. M.; Cosper, S. W.; Hyde, E. K.; Phys. Rev. Lett. 1966, 17, 1271; Tanihata, I.; Kanungo, R.; C. R. Physique 4 2003, 437; Tanihata, I.; Hamagaki, H.; Hashimoto, O.; Nagamiya, S.; Shida, Y.; Yoshikawa, N.; Yamakawa, O.; Sugimoto, K.; Kobayashi, T.; Greiner, D. E.; Takahashi, N.; Nojiri, Y.; Phys. Lett. B 1985, 160, 380.

7. Gamow, G.; Birth And Death Of The Sun, $1^{\text {st }}$ ed., Dover Publications: New York, 2005; Silk, J.; O Big Bang, 2a ed., Editora UnB: Brasília, 1988.

8. Stoyer, M. A.; Nature 2006, 442, 876.

9. Azhari, A.; Kryger, R. A.; Thoennessen, M.; Phys. Rev. C 1998, 58, 2568; Leite, T. N.; Teruya, N.; Dimarco, A.; Duarte, S. B.; Tavares, O. A. P.; Gonçalves, M.; Phys. Rev. C 2009, 80, 014606.

10. Tanihata, I.; Hirata, D.; Kobayashi, T.; Shimoura, S.; Sugimoto, K.; Toki, H.; Phys. Lett. B 1992, 289, 261; Teruya, N.; Bertulani, C. A.; Krewald, S.; Dias, H.; Hussein, M. S.; Phys. Rev. C 1991, 43, R2049; Bertulani, C. A.; Canto, L. F.; Hussein, M. S.; Physics Reports 1993, 226, 281.

11. Warner, D.; Nature 2004, 430, 517.

12. Kemper, K. W.; Cottle, P. D.; Physics 2010, 3, 13; disponível em http:// physics.aps.org/articles/v3/13, acessada em Janeiro 2011.

13. Sagawa, H.; Suzuki, T.; Nucl. Phys. A 2001, 687, 111c; Dang, N. D.; Au, V. K.; Suzuki, T.; Arima, A.; Phys. Rev. C 2001, 63, 044302.; Leite , T. 
N.; Teruya, N.; Eur. Phys. J. 2004, 21, 369; Nakamura, T.; Vinodkumar, A. M.; Sugimoto, T.; Aoi, N.; Baba, H.; Bazin, D.; Fukuda, N.; Gomi, T.; Hasegawa, H.; Imai, N.; Ishihara, M.; Kobayashi, T.; Kondo, Y.; Kubo, T.; Miura, M.; Motobayashi, T.; Otsu, H.; Saito, A.; Sakurai, H.; Shimoura, S.; Watanabe, K.; Watanabe, Y. X.; Yakushiji, T.; Yanagisawa, Y.; Yoneda, K.; Phys. Rev. Lett. 2006, 96, 252502.

14. Galetti, D.; Lima, C. L.; Energia Nuclear com fissões e com fusões, $1^{\mathrm{a}}$ ed., Editora Unesp: São Paulo, 2010.

15. Bethe, H. A.; Phys. Rev. 1939, 55, 434; Gamow, G.; Phys. Rev. 1946, 70, 572; Alpher, R. A.; Bethe, H.; Gamow, G.; Phys. Rev. 1948, 73, 803; Alpher, R. A.; Herman, R. C.; Reviews of Modern Physics 1950, 22, 153.
16. Burbidge, E. M.; Burbidge, G. R.; Fowler, W. A.; Hoyle, F.; Reviews of Modern Physics 1957, 29, 547.

17. Duarte, S. B.; Dissertação de Mestrado, Centro Brasileiro de Pesquisas Físicas, Brasil, 1977; Hillebrand, W.; Takarashi, K.; Kodama, T.; Astronomy \& Astrophysics 1976, 52, 63.

18. Qian, Y. Z.; Progress in Particle and Nuclear Physics 2003, 50, 153; Sneden, C.; Cowan, J. J.; Science 2003, 299, 70. 